Article

\title{
Exploring No-Cost Opportunities for Public Sector Information Systems Energy Efficiency: A Tennessee Application
}

\section{Kendra Abkowitz Brooks ${ }^{1, *}$ and Talton Pettigrew ${ }^{2}$}

1 Office of Policy and Planning, Tennessee Department of Environment and Conservation, William R. Snodgrass Tennessee Tower, 312 Rosa L. Parks Avenue, 2nd Floor, Nashville, TN 37243, USA

2 Information Services Division, William R. Snodgrass Tennessee Tower, 312 Rosa L. Parks Avenue, 2nd Floor, Nashville, TN 37243, USA; E-Mail: talton.pettigrew@tn.gov

* Author to whom correspondence should be addressed; E-Mail: Kendra.abkowitz@tn.gov; Tel.: +1-615-532-8689.

Academic Editor: Richard Matthew

Received: 28 August 2015 / Accepted: 28 October 2015 / Published: 3 November 2015

\begin{abstract}
The Tennessee Department of Environment and Conservation (TDEC) completed a pilot project within its Central Office spaces to test the utilization of computer power management (CPM) technologies to implement power saving settings on state-owned, network-connected computer equipment. Currently, the State of Tennessee has no clear protocol regarding energy-conserving power settings on state-owned machines. Activation of monitor sleep modes and system standby and hibernation modes on 615 Central Office computers over an 18-month period reduced energy consumption by an estimated $8093 \mathrm{kWh}$ and \$526 per month, amounting to approximately $\$ 6312$ in cost savings for Tennessee annually. If implemented throughout State of Tennessee executive agencies across the state, energy cost savings could amount to an estimated $\$ 323,341$ annually. The research endeavored to understand both positive and negative impacts that strategic power management approaches can have on energy consumption, worker productivity, network security, and state budgets. Nearly all impacts discussed were positive. Based on successful results within TDEC Central Office spaces in Tennessee Tower, and considering the potential cost savings that could be achieved, expansion of the implementation of computer power management policies to machines in offices across the state was recommended.
\end{abstract}

Keywords: computer energy management; public sector energy efficiency; public sector computer energy consumption; public sector sustainability; computer energy conservation 


\section{Introduction}

As our technology within society has become increasingly advanced and commonplace, work environments have seen an increase in computing equipment, which requires generation and consumption of electricity for operation. While computers and accessories have become increasingly efficient, opportunities for reducing energy consumption through computer power management programs still exist, which can translate into avoiding considerable financial and environmental costs. Previous research notes the opportunity for computer power management to provide savings based on modeling projections; however, few studies document actual energy and cost savings achieved through computer power management programs, specifically within a larger sample size and within public sector workplaces over an extended period of time. This research attempts to answer the following questions: how effective are computer power management programs when implemented throughout a public sector organization, over an extended period of time, and what kinds of positive and negative quantitative and qualitative impacts may result from implementation?

The Tennessee Department of Environment and Conservation (TDEC), in collaboration with the Department of Finance and Administration's Office for Information Resources (OIR), completed a pilot project within its Central Office spaces to test the utilization of computer power management (CPM) technologies to implement power saving settings on state-owned, network-connected computer equipment. The intent of this effort is to better understand both the positive and negative impacts that strategic power management approaches can have on energy consumption, worker productivity, network security, and state budgets. Currently, the State of Tennessee has no clear protocol regarding energy-conserving power settings on state-owned machines. While power saving settings are available on state-owned electronics, these settings must be activated on each individual machine, and can easily be superseded by the user. Currently, users are encouraged to leave their computers on overnight so that updates can be received.

At the outset of this study, TDEC had an estimated 1555 desktop machines and 345 laptop machines (1900 total) across the state. Within its Central Office and at the initiation of the project, TDEC was operating and maintaining approximately 800 machines. Activation of monitor sleep modes and system standby and hibernation modes on a 12-machine subset of TDEC Central Office computers over a 53-day period (CPM mini-pilot), saved the department approximately \$17. Based on savings achieved during the CPM mini-pilot, if power management policies (A power management policy refers to power management settings that administrators can create to define power management configurations, such as specified times for sleep modes and wake for updates, for groups of users and/or computers.) were activated on all TDEC Central Office computers, it was calculated that approximately $\$ 7500$ could be saved annually. Due to the potential savings indicated during the CPM mini-pilot, TDEC recommended expansion of the power management policy to its Central Office spaces throughout the remainder of 2014 and early 2015 to allow for potential positive and negative impacts of such implementation to be monitored and evaluated. This expansion has been completed such that a total of approximately 615 machines within TDEC's Central Office now have power management savings activated (During Spring/Summer 2015, ISD deployed tablets that replaced a considerable number of desktops and laptops. Additionally, due to the need to remote access machines and for other special circumstances, 30 computers are excluded from the policy. TDEC investigated 
whether or not the decrease in machines was attributed to a commensurate decrease in TDEC Central Office employees. According to TDEC-Human Resources reports, the number of Central Office employees has increased by a net of 13 individuals from January 2014 to May 2015. Therefore, the decrease in number of Central Office machines cannot be directly attributed to a decrease in Central Office Staff.). Based on results through the end of May 2015, computer power management policies within TDEC Central Office spaces are reducing energy consumption by an estimated $8093 \mathrm{kWh}$ and $\$ 526$ per month. This amounts to approximately \$6312 in cost savings annually, attributed to reduced energy consumption that has resulted from the implementation of computer power management on TDEC Central Office computers. If implemented on computers at all of TDEC's field locations, energy cost savings could amount to an estimated \$16,937 annually (Assumes approximately 1650 TDEC computers capable of being managed by computer power management policies.). If implemented throughout State of Tennessee executive agencies across the state, energy cost savings could amount to an estimated \$323,341 annually (Assumes approximately 31,500 computers statewide overseen by OIR and capable of being managed by computer power management policies.). Based on successful results within the TDEC Central Office spaces in Tennessee Tower, and considering the potential cost savings that could be achieved, expansion of the implementation of computer power management policies to machines in offices across the state was recommended.

The paper documenting TDEC's efforts is organized as follows. First a brief review of the state of the practice of computer power management is provided. Then the research methodology utilized for implementation is discussed, followed by an analysis and discussion of results achieved during the research project. Finally, future opportunities for additional research, recommendations, and conclusions are presented in the last section.

This research serves as a supplement to previous research completed by the authors regarding pilot project progress and supplements the original Computer Power Management Pilot Proposal (Abkowitz Brooks and Pettigrew, 2014) [1] and Status Reports (Abkowitz Brooks and Pettigrew, 2015) [2].

\section{State of the Practice of Computer Power Management}

Utilization of modern information technology has become a necessity for a functioning 21 st century workplace. The influx of computing solutions and accessibility to these solutions through mobile technology has flooded work environments across the globe with electricity-consuming equipment. However, the widespread incorporation of computers and their peripherals require electricity to operate, and this electricity must be generated from resources and, consequently, distributed to work environments through transmission and distribution systems. While electricity allows for a highly functional and efficient society, its generation has considerable financial and environmental costs (Pickavet et al., 2008) [3]. According to the U.S. Energy Information Administration (EIA), the average retail price of electricity for the commercial sector in 2014 amounted to 10.75 cents per $\mathrm{kWh}$ (EIA, 2015) [4]. Based on this average cost, a typical desktop computer with dual LCD monitors would cost just over $\$ 40$ annually to operate (Assumes a desktop computer which uses 100 watts and two LCD monitors operating $8 \mathrm{~h}$ a day for 252 days a year.). While this might not seem like a significant expense, multiplied by the hundreds or thousands, it becomes a more sizable operating expense for larger organizations, such as universities, major corporations, and federal, state, and local 
governments (Pickavet et al., 2008) [3]. For public sector work environments in particular, the combination of increasing pressure to utilize taxpayer dollars wisely and transparently, historical delays in the adoption of cutting edge technology solutions compared to the private sector, and dwindling budgets for administrative operations provide a real opportunity for computer power management protocols to become an easily-implementable and low or no-cost way to reduce operating expenses (Alam and Brooks, 2014) [5] and (Alam and Brooks, 2013) [6].

It should be noted, however, that over time, the efficiency of computers has increased dramatically. As the footprint associated with computers has grown in recent years, the industry has responded with development of increasingly energy efficient information technology products and has sought to configure them in ways which reduce the amount of energy required to operate. Nearly all computer operating systems are now equipped with power saving settings, but the extent to which these are activated and utilized is relatively unclear. Despite all this, there are still ample opportunities for reducing energy consumption within work environments, particularly within the slower-to-adopt public sector. Agarwal et al. separate information technology energy management into three categories (Agarwal et al., 2010) [7]:

(1) Reducing the active power consumption of individual computing devices by utilizing lower power components or using them more efficiently.

(2) Migrating work between machines - to consolidate onto a smaller number of servers or to arbitrage advantageous energy prices in different geographic zones.

(3) Duty-cycling subsystems during periods of idleness or low use.

Research regarding computer energy consumption has historically focused on the first and third categories described by Agarwal et al. [7] above. Nedevschi et al. in particular discuss opportunities to utilize network improvements to achieve energy savings. The authors note that networks are designed to be capable of delivering the maximum load possible, not what typically is required for sufficient and long-term operation. Further energy consumption of equipment connected to the network continues at sometimes significant levels despite the network itself being inactive (Nedevschi et al., 2008) [8] (The authors go so far to indicate that most of the energy consumed in networks is wasted.). This leads to the conclusion that there still remains much to be done in terms of using networks which connect computers and peripherals to be used more effectively to efficiently manage computer energy consumption.

Implementation of a proxy scheme that allows end hosts to utilize sleep modes to save energy, without forfeiting network connectivity or availability have become increasingly common. "Wake-on-LAN" technology has enabled systems in a low-power state after a long period of idling, or sleeping, to be awakened once a signal or special packet has been delivered through the network to the machine. However, one of the challenges within this framework is determining the proper time for networks to send this packet to wake computers such that it maintains its energy saving purposes without inconveniencing the user (Agarwal et al., 2010) [7]. A software product that utilizes this approach, SleepServer, has been shown to result in energy savings ranging from $27 \%$ to $86 \%$ among a sample of thirty desktop users (Agarwal et al., 2010) [7]. Likewise, proxying, application-specific wake-up, and split transmission control protocol (TCP) connections have been shown to increase the 
low-power sleep time of personal computers (PCs) in amounts of around $\$ 2.7$ billion per year if implemented across the U.S. (Gunaratne et al., 2005) [9].

Despite the ample research available that power management options can result in energy efficiency improvements and cost savings, few studies provide documentation of actual energy and cost savings achieved through computer power management programs. Rather, the vast majority of research discusses modeling outputs illustrating the potential results of energy savings. For those studies which do include empirical data, it reflects savings achieved through implementation within a small subset of machines over a relatively short period of time. Therefore, research available to document energy and cost savings achievable in public sector workplaces over an extended period of time is limited. This study aims to serve as a proof of concept regarding opportunities for public sector cost savings through computer power management policies.

\section{Central Office Implementation Methodology}

In 2013, an interdisciplinary group of professionals within TDEC was tasked with identifying low- and no-cost opportunities within the agency for reducing environmental impact and lowering operating costs. One of the recommendations the group provided to departmental leadership was adoption of clear instructions regarding computer power management and, further, investigation of opportunities to centrally implement computer power management throughout networked computers. To accomplish this end, TDEC staff conducted research regarding software solutions available within the marketplace and utilized by organizations similar in size to deploy power saving settings across networked computers. TDEC discovered several different solutions which ranged in cost and capabilities significantly. However, during the course of this research, TDEC discovered that its current systems management software, Microsoft System Center Configuration Manager (SCCM), had the capability of distributing power saving settings among networked machines (Microsoft) [10,11]. TDEC had previously never utilized this feature of SCCM. Given the project's focus on low- or no-cost implementation, the project team decided to utilize the existing systems management software in lieu of other solutions available on the market.

Prior to implementing on a grand scale, two different policy schemes were designed and tested within a small subset of 12 computers (mini-pilot). Based on results of the mini-pilot indicating actual, measured energy savings with minimal impact to users based on the testing of various policies, TDEC recommended that power management Policy B be activated within its Central Office utilizing a phased approach where subsets of floors are impacted (During previous phases of research, multiple power management policies were evaluated to determine appropriateness for implementation within the pilot. Reference unpublished research by Abkowitz Brooks and Pettigrew, which can be obtained by contacting the authorities.). Under Policy B, as originally implemented, during all hours, monitor sleep modes were activated after 5 min of inactivity and machine sleep modes were activated after 10 min of inactivity between the hours of 4:00 p.m. and 5:00 a.m., hours during which employees are typically out of the office. Computers were woken for updates daily between 5:30 and 6:30 a.m., prior to typical working hours. 
Following initial rollout of the power management Policy B to second-floor machines, feedback received from the second floor user survey indicated that slight modifications in the policy were necessary - particularly that users felt as though computers were placed in sleep mode too quickly.

On 15 May 2014, the power management policy was revised such that during all hours, monitor sleep modes were activated after 15 min of inactivity. Between the hours of 4:30 p.m. and 5:00 a.m. (non-work hours), machine sleep modes were activated after $30 \mathrm{~min}$ of inactivity. Computers are woken for updates daily between 5:30 and 6:30 a.m. Once these modifications were made, follow-up feedback regarding the change in policy was positive.

Policies were implemented by floor as follows by a single TDEC ISD staff person:

- 2nd floor implementation (13 March 2014) and user feedback survey (24 April 2014)

- 14th and 15th floor implementation (Power management policy implementation for 8 computers on the 3rd floor was also included during this phase.) (2 June 2014) and user feedback survey (19 June 2014)

- 10th, 11th, and 12th floor implementation (14 July 2014) and user feedback survey (4 August 2014)

Prior to each phase of implementation, ISD distributed a notification email informing users of power management policy implementation, and providing users who would like to be excluded from the policy due to impacted services, such as remote desktop connection, to opt out of policy inclusion. As an example, the text of one of those messages is provided below.

"All,

On Monday, July 14, we will roll out minor power management changes to all computers on the 10th, 12th, and 14th floors of the Tennessee Tower. We are following a successful 3 month testing/tweaking phase on the 2 nd floor, and we are confident these settings provide optimal energy savings while having a minimal impact on our staff. While we don't expect an impact on what you are familiar with, please keep the following in mind:

- During the daytime hours computer monitors are going to go to 'sleep' after 15 min of inactivity. The screens will turn black. All that is needed to 'wake' them is movement of your mouse or pressing a key on your keyboard.

- Overnight, the computers themselves will go to sleep (this may affect a few services for very limited number of people, listed below) after $30 \mathrm{~min}$ of inactivity and will be scheduled to wake back up before the daytime hours begin. If your computer is still asleep when you come in for your workday, click a mouse button or press a keyboard key and wait a few seconds to wake it. If this doesn't work simply press the power button on your computer.

- 'Daytime hours' for this purpose are 5:00 a.m. to 4:30 p.m. Saturday and Sunday follow the same schedule, so if the services listed below are needed on the weekend but only during those hours-notifying me is not required.

- Also note: it is still state policy to leave your computer at the log in screen over night, so you will still want to 'restart' your computer at the end of the work day and leave it running.

- Services affected by a computer going to sleep over night: 
(1) Remote Desktop access: if you remote into your work computer outside of daytime hours, please let me know so I can ensure you are set up correctly.

(2) Polling: If you have a computer that polls any outside service overnight or every hour and needs to be 'on' all the time, please let me know so I can ensure you are set up correctly.

(3) Any script that runs outside of daytime hours: For example, backup scripts to external hard drives, or replications for database developments. These would fail to run if the computer were asleep so we will need to set you up differently or set these up to run at a different time.

Please let me know if you believe you fall under one of the affected services, by Monday, July 14th at 8 a.m. We will then make sure your computer is set up correctly for this new policy. Any other feedback, questions or concerns regarding this testing should be sent to both Kendra Abkowitz (Kendra.abkowitz@tn.gov) and me (talton.pettigrew@tn.gov).”

\section{Results and Discussion}

Results from the pilot project through the end of May 2015 will now be discussed.

\subsection{ISD Staff and User Accessibility}

The power management policy implemented has continued to provide users the convenience of keeping machines awake throughout the day, while allowing continued ease of access for ISD technicians to perform work during early morning hours. As previously mentioned, extending inactivity criteria in the policy for monitor sleep modes from 5 to 15 min during work hours addressed feedback regarding slight inconveniences that were voiced by TDEC employees.

Additionally, due to the early notification of power management policy implementation that was distributed to TDEC employees, users were informed of changes and were given an opportunity to notify ISD if exclusion or alternative policy implementation was necessary. This proactive approach not only addressed potential concerns, it also served as education regarding the power management policy implementation. Surveys requesting user feedback were distributed to impacted floors two to six weeks following the power management policy implementation. Survey responses were instrumental in identifying any specific issues or concerns that may have resulted from the power management policies, but also prompted users to seek assistance from ISD for other issues or concerns that they thought were related to the power management policy, that in reality were unrelated. The overwhelming majority of responses were positive.

\subsection{Network Bandwidth}

After observing no identifiable changes in bandwidth impacts during the mini-pilot, as part of the original pilot project proposal, TDEC requested monthly reports from OIR concerning negative bandwidth impacts to Tennessee Tower machines, as well as the entire network. However, monthly reports have not been received from OIR. TDEC did reach out to OIR in September 2014 to gauge whether or not there had been any identifiable changes in bandwidth or network impacts that could be 
tied to the power management pilot. No response was received from OIR. TDEC reached out to OIR again regarding any noticeable impacts in January 2015 and June 2015. On both occasions, OIR, after reviewing bandwidth logs, indicated that the bandwidth impact on the network was negligible, but that it could not be ascertained if negligibility of impacts was due to the presence on the metro area network and/or size of sample utilized within the study (As indicated via email from Alan Atherton, 23 January 2015 and 10 June 2015, respectively. Atherton also indicated: "If a large building with high user count or a site with smaller bandwidth uses the energy savings settings there could be latency experienced by the end site. We would have to monitor to ascertain that (January 2015)". "Most network issues around bandwidth would be negated by your presence on the MAN [metro area network] and the relatively small footprint of devices participating in the power study. The number one issue we have with staff that are 'off-network' or turn their devices off at night is the impact of downloading software operating system and virus pattern client updates when they power up their computers (June 2015)”).

\subsection{Energy Consumption}

Daily energy consumption for computers included in the pilot project was logged each day by SCCM (In order to report energy consumption, a computer must be powered on and on the network. There it normally some minimal fluctuation between number of computers reporting from one day to the next. On rare occasions more significant fluctuations have been noticed.). SCCM then aggregated daily energy consumption by floor for each month to calculate an average monthly energy consumption per computer. These average energy consumption numbers are those which are noted throughout this report. The following Tables 1 and 2 and Figures 1 and 2 serve as illustrative examples of reductions in computer energy consumption for select floors based on report exports from SCCM during the pilot project (Results on a floor-by-floor basis are available but have been excluded from the article due to their repetitive nature. A summary of savings for each floor is provided in following Sections).

Compared to no power management policy, following implementation of Policy B, monthly average energy consumption by computer for second-floor devices dropped approximately $8.19 \mathrm{kWh}$, from an average of 23.99 to an average of $15.80 \mathrm{kWh}$. This represents a $34 \%$ decrease in energy consumption compared to the no power management scenario. Similarly, compared to no power management policy, following implementation of Policy B, monthly average energy consumption by computer for 10th-floor devices dropped approximately $14.19 \mathrm{kWh}$, from an average of 43.04 to an average of $28.85 \mathrm{kWh}$. This represents a $33 \%$ decrease in energy consumption compared to the no power management scenario. 
Table 1. Second-floor computer energy consumption.

\begin{tabular}{cccc}
\hline & & 2nd FLOOR & \\
\hline & $\begin{array}{c}\text { Monthly Average Energy } \\
\text { Consumption by Computer (kWh) }\end{array}$ & Policy Treatment & $\begin{array}{c}\text { Average Monthly Energy } \\
\text { Consumption per Computer (kWh) }\end{array}$ \\
\hline Jan 2014 & 24.17 & No Power Management & 23.99 \\
Feb 2014 & 23.80 & & \\
\hline Mar 2014 & 18.06 & & \\
Apr 2014 & 12.98 & & \\
May 2014 & 15.09 & & \\
June 2014 & 15.43 & & \\
July 2014 & 16.74 & \\
Aug 2014 & 15.27 & & \\
Sept 2014 & 15.66 & & \\
Oct 2014 & 16.93 & \\
Nov 2014 & 14.73 & & \\
Dec 2014 & 16.19 & \\
Jan 2015 & 16.69 & \\
Feb 2015 & 15.40 & \\
Mar 2015 & 16.23 & & \\
Apr 2015 & 16.10 & & \\
May 2015 & 15.45 & \\
\hline
\end{tabular}

\section{2nd Floor Average Energy Consumption by Computer (kWh)}

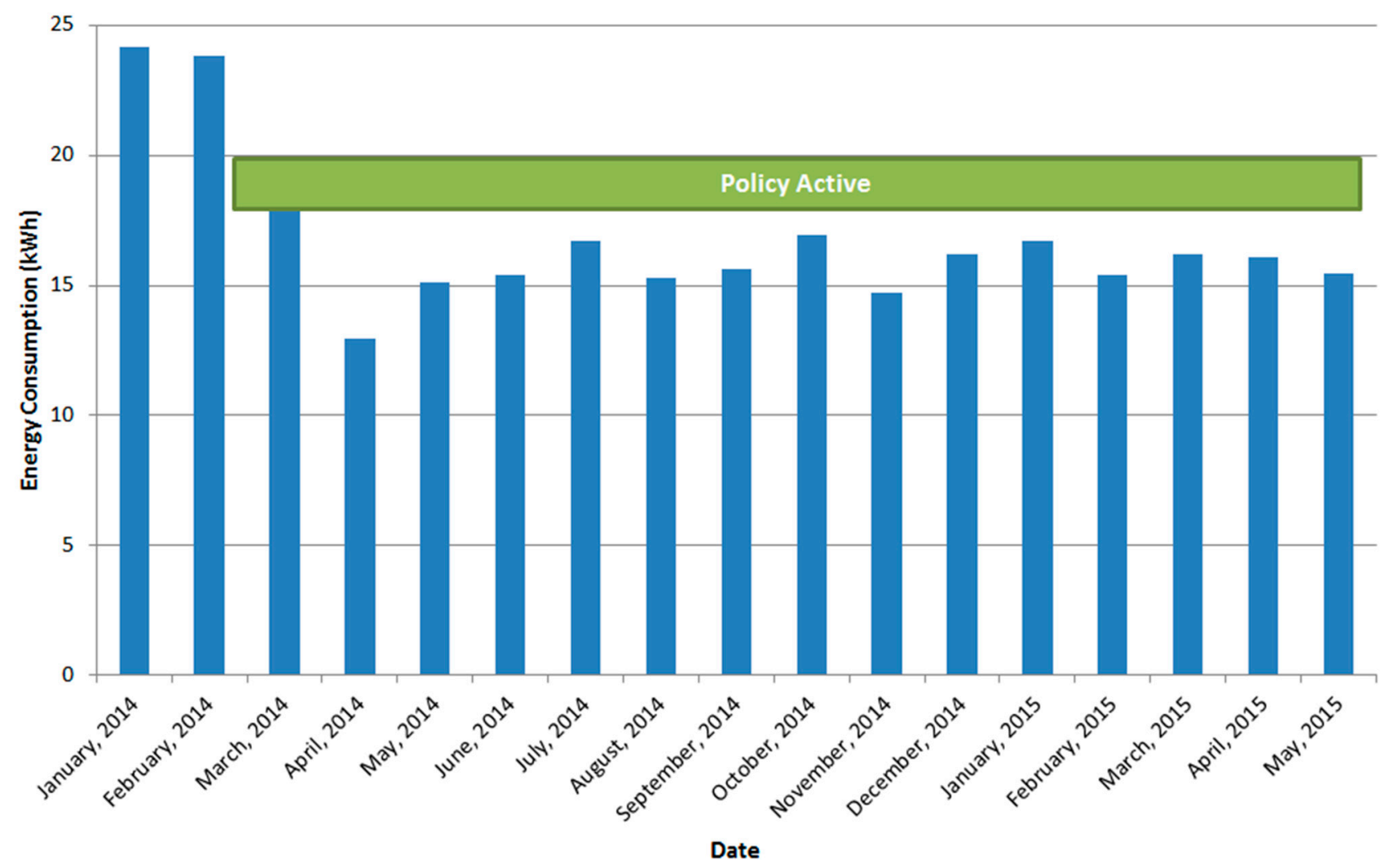

Figure 1. Second-floor average energy consumption by computer (kWh). 
Table 2. 10th-floor computer energy consumption.

\begin{tabular}{cccc}
\hline & & 10th FLOOR & \\
\hline & $\begin{array}{c}\text { Monthly Average Energy } \\
\text { Consumption by Computer (kWh) }\end{array}$ & Policy Treatment & $\begin{array}{c}\text { Average Monthly Energy } \\
\text { Consumption per Computer (kWh) }\end{array}$ \\
\hline Jan 2014 & 48.45 & & \\
Feb 2014 & 43.82 & & \\
Mar 2014 & 25.99 & No Power Management & \\
Apr 2014 & 48.04 & & \\
May 2014 & 47.43 & & \\
June 2014 & 44.48 & & \\
\hline July 2014 & 34.11 & & \\
Aug 2014 & 21.95 & & \\
Sept 2014 & 26.27 & & \\
Oct 2014 & 31.68 & & \\
Nov 2014 & 29.04 & & \\
Dec 2014 & 29.98 & \\
Jan 2015 & 29.40 & & \\
Feb 2015 & 25.16 & & \\
Mar 2015 & 28.28 & & \\
Apr 2015 & 31.83 & & \\
May 2015 & 29.60 & & \\
\hline & Estimated Energy Savings by Computer (kWh) & \\
\hline
\end{tabular}

10th Floor Average Energy Consumption by Computer (kWh)

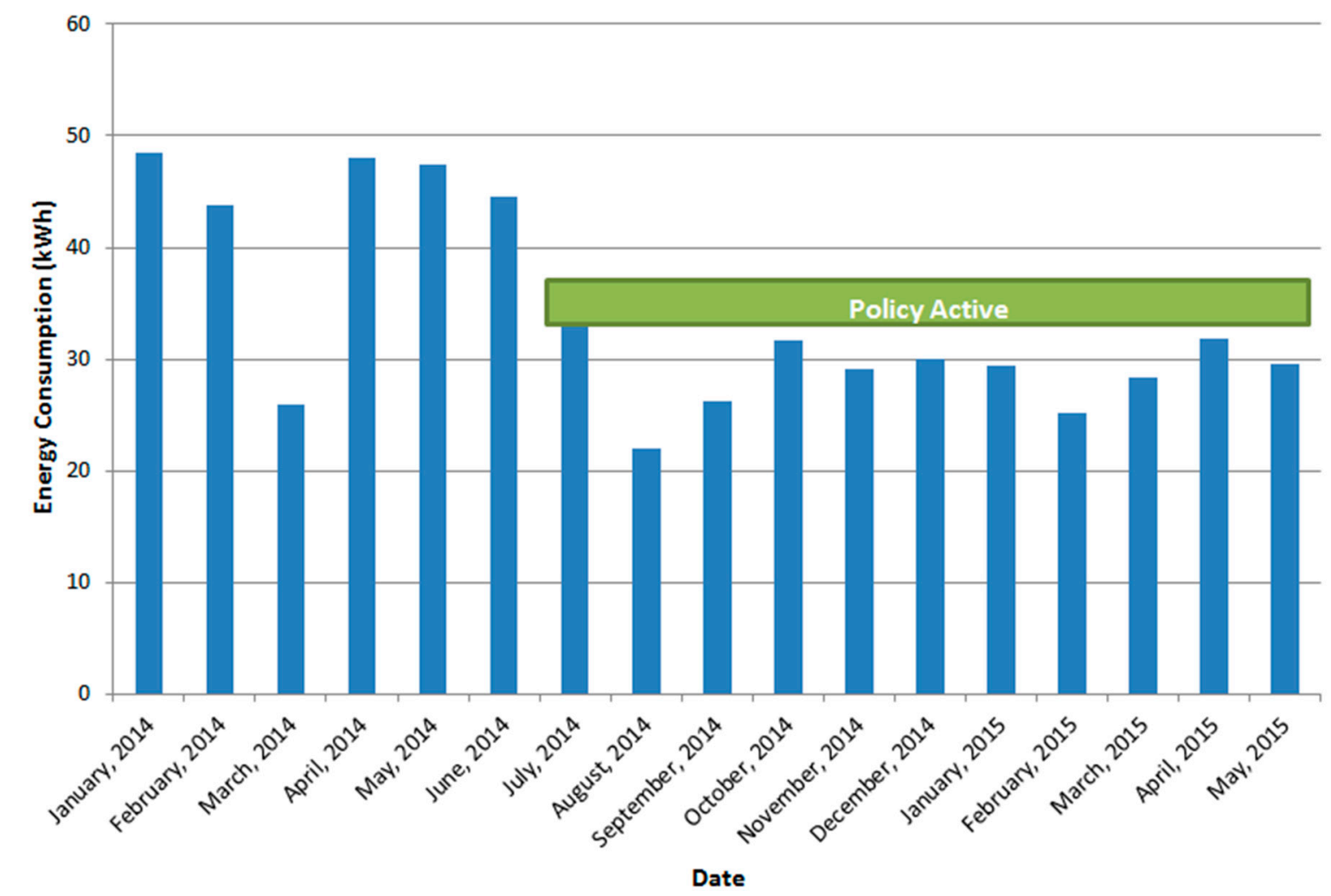

Figure 2. 10th floor average energy consumption by computer $(\mathrm{kWh})$. 


\subsection{Energy Costs}

According to the Department of General Services (DGS) State of Tennessee Real Estate Asset Management (STREAM) group, energy costs amount to approximately $\$ 0.065$ per $\mathrm{kWh}$ within William R. Snodgrass Tennessee Tower (Tennessee Tower). Since all computers that are part of the pilot project operate within Tennessee Tower, a $\$ 0.065$ per $\mathrm{kWh}$ cost rate was utilized when determining energy costs. Using the average energy consumption per computer per month for each floor, before and after the power management policy implementation as described in the previous Energy Consumption section and the $\$ 0.065$ per kWh energy cost rate provided by DGS, a monthly average energy consumption cost per computer was calculated. The following Tables 3 and 4 and Figures 3 and 4 are an illustrative example of estimated reductions in computer energy cost based on energy consumption reports exported from SCCM before and after implementation of the computer power management policy (Results on a floor-by-floor basis are available but have been excluded from the article due to their repetitive nature. A summary of savings for each floor is provided in following sections.).

Table 3. Second-floor computer energy costs.

\begin{tabular}{cccc}
\hline \multicolumn{3}{c}{ 2nd FLOOR } \\
\hline & $\begin{array}{c}\text { Monthly Average Energy } \\
\text { Cost per Computer (\$) }\end{array}$ & Policy Treatment & $\begin{array}{c}\text { Average Monthly Energy } \\
\text { Cost per Computer (\$) }\end{array}$ \\
\hline Jan 2014 & 1.57 & No Power Management & 1.56 \\
Feb 2014 & 1.55 & & \\
\hline Mar 2014 & 1.17 & & \\
Apr 2014 & 0.84 & & \\
May 2014 & 0.98 & \\
June 2014 & 1.00 & \\
July 2014 & 1.09 & \\
Aug 2014 & 0.99 & \\
Sept 2014 & 1.02 & \\
Oct 2014 & 1.10 & \\
Nov 2014 & 0.96 & \\
Dec 2014 & 1.05 & \\
Jan 2015 & 1.08 & \\
Feb 2015 & 1.00 & \\
Mar 2015 & 1.05 & \\
Apr 2015 & 1.05 & \\
May 2015 & 1.00 & \\
\hline Estimated Monthly Energy Cost Savings per Computer (\$) & \\
\hline
\end{tabular}




\section{2nd Floor Estimated Average Monthly Energy Cost per Computer (\$)}

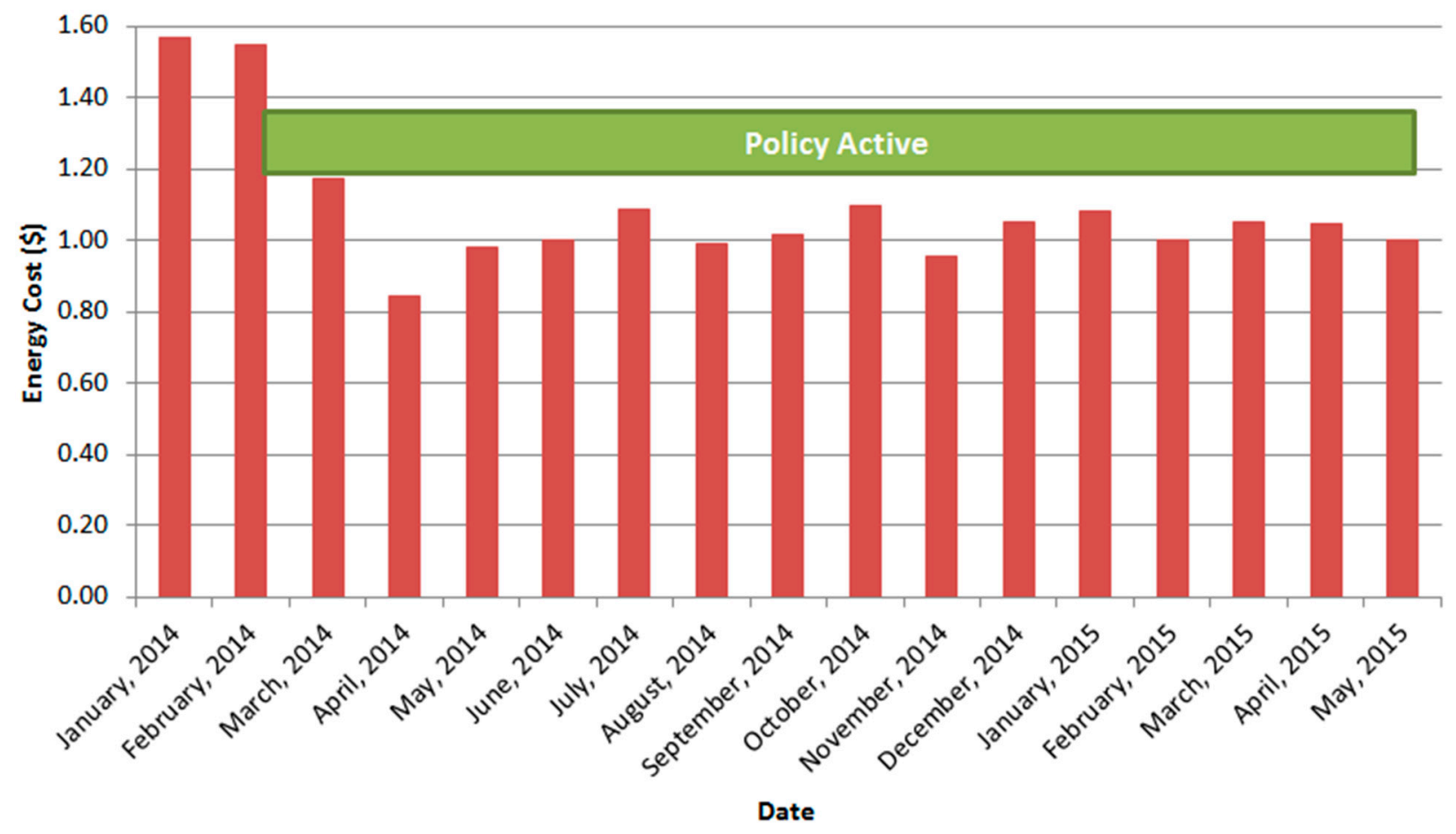

Figure 3. Second-floor average monthly energy cost per computer (\$).

Compared to no power management policy, following implementation of power management Policy B, monthly average energy cost by computer for second-floor devices dropped approximately $\$ 0.53$, from $\$ 1.56$ to $\$ 1.03$. This represents a $34 \%$ decrease in energy costs relative to the no power management scenario. Similarly, compared to no power management policy, following implementation of power management Policy B, monthly average energy cost by computer for 10th-floor devices dropped approximately $\$ 0.92$, from $\$ 2.80$ to $\$ 1.88$. This represents a $33 \%$ decrease in energy costs relative to the no power management scenario.

Table 4. 10th-floor computer energy costs.

\begin{tabular}{cccc}
\hline \multicolumn{4}{c}{ 10th FLOOR } \\
\hline & $\begin{array}{c}\text { Monthly Average Energy } \\
\text { Cost per Computer (\$) }\end{array}$ & Policy Treatment & $\begin{array}{c}\text { Average Monthly Energy } \\
\text { Cost per Computer (\$) }\end{array}$ \\
\hline Jan 2014 & 3.15 & & \\
Feb 2014 & 2.85 & & \\
Mar 2014 & 1.69 & No Power Management & \\
Apr 2014 & 3.12 & & \\
May 2014 & 3.08 & & \\
June 2014 & 2.89 & & \\
\hline
\end{tabular}


Table 4. Cont.

\begin{tabular}{cccc}
\hline \multicolumn{3}{c}{ 10th FLOOR } \\
\hline & $\begin{array}{c}\text { Monthly Average Energy } \\
\text { Cost per Computer (\$) }\end{array}$ & Policy Treatment & $\begin{array}{c}\text { Average Monthly Energy } \\
\text { Cost per Computer (\$) }\end{array}$ \\
\hline July 2014 & 2.22 & \\
Aug 2014 & 1.43 & \\
Sept 2014 & 1.71 & \\
Oct 2014 & 2.06 & \\
Nov 2014 & 1.89 & \\
Dec 2014 & 1.95 & \\
Jan 2015 & 1.91 & \\
Feb 2015 & 1.64 & \\
Mar 2015 & 1.84 & \\
Apr 2015 & 2.07 & \\
May 2015 & 1.92 & \\
\hline Estimated Monthly Energy Cost Savings per Computer (\$) & \\
\hline
\end{tabular}

10th Floor Estimated Average Monthly Energy Cost per Computer (\$)

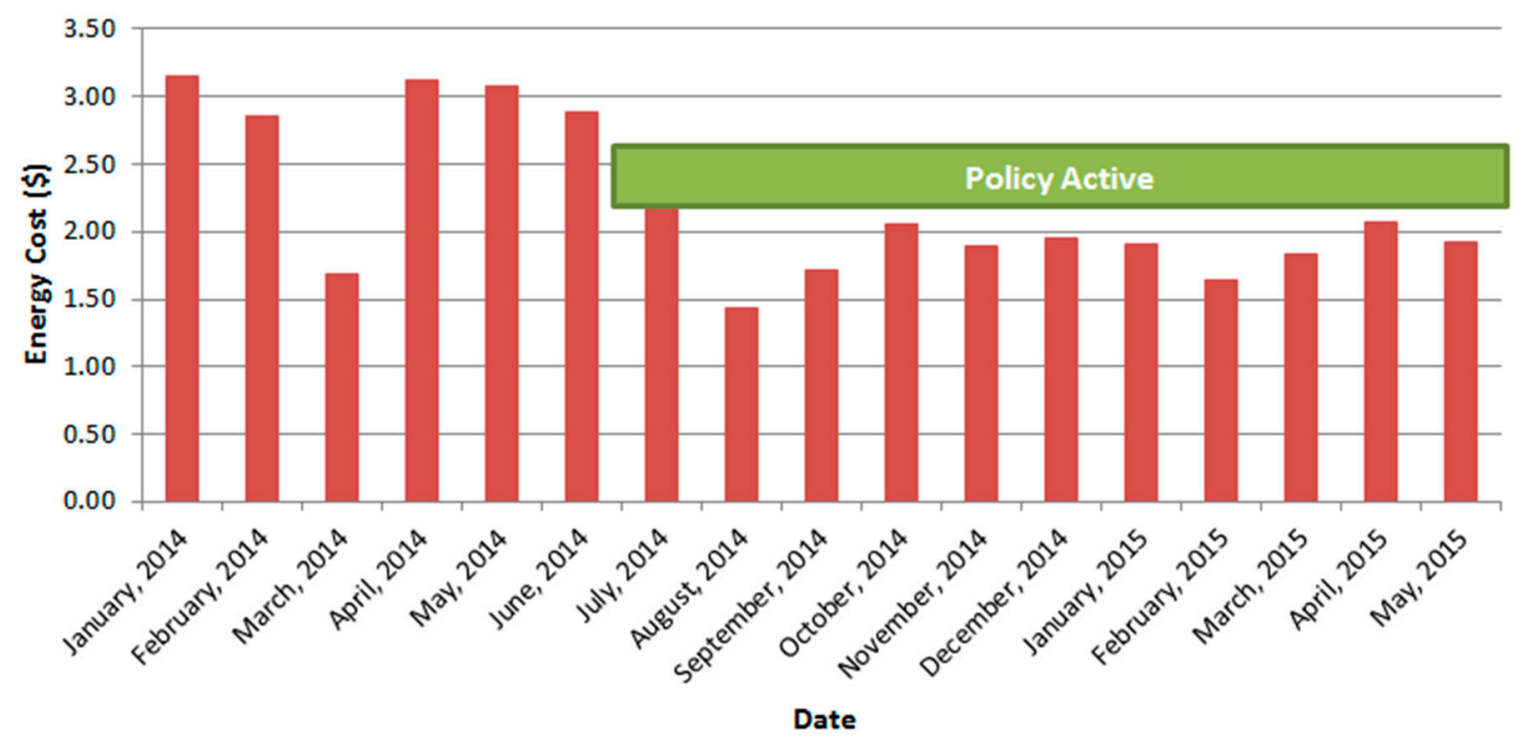

Figure 4. 10th-floor average monthly energy cost per computer $(\$)$.

Floors 11, 12, 14, and 15 experienced similar energy cost savings results as a result of implementation of Policy B compared to no power management:

- Monthly average energy cost by computer for 11 th-floor devices dropped approximately $\$ 0.40$; from $\$ 1.70$ to $\$ 1.29$, a $24 \%$ decrease in energy costs relative to the no power management scenario.

- Monthly average energy cost by computer for 12 th-floor devices dropped approximately $\$ 0.74$, from $\$ 2.48$ to $\$ 1.74$, a $30 \%$ decrease in energy costs relative to the no power management scenario.

- Monthly average energy cost by computer for 14th-floor devices dropped approximately $\$ 0.92$, from $\$ 2.46$ to $\$ 1.54$, a $37 \%$ decrease in energy costs relative to the no power management scenario. 
- Monthly average energy consumption by computer for 14th-floor devices dropped approximately $14.20 \mathrm{kWh}$, from an average of 37.92 to an average of $23.71 \mathrm{kWh}$, a $37 \%$ decrease in energy consumption compared to the no power management scenario.

- Monthly average energy cost by computer for 15th-floor devices dropped approximately $\$ 1.61$, from $\$ 2.67$ to $\$ 1.06$, a $60 \%$ decrease in energy costs relative to the no power management scenario.

To determine average energy savings and cost savings across all TDEC Central Office floors, because power management policies were implemented at different times, the averages of monthly energy consumption by computer under the no power management and power management scenarios for all floors was calculated. The difference between no power management and power management scenarios average was determined to represent monthly average energy consumption savings by computer for all of TDEC Central Office. This number was then multiplied by the number of Central Office computers to estimate total monthly energy savings for all computers with active power management settings. Table 5 below illustrates the data and calculations utilized to determing Central Office computer power management energy and cost savings.

Table 5. TDEC Central Office computer power management energy and cost savings.

\begin{tabular}{|c|c|c|c|}
\hline & Floor & No Power Management & Power Management \\
\hline \multirow{7}{*}{$\begin{array}{l}\text { Monthly Average Energy Consumption by } \\
\text { Computer }(\mathrm{kWh})\end{array}$} & 2 & 23.99 & 15.80 \\
\hline & 10 & 43.04 & 28.85 \\
\hline & 11 & 26.11 & 19.90 \\
\hline & 12 & 38.17 & 26.76 \\
\hline & 14 & 37.92 & 23.71 \\
\hline & 15 & 41.04 & 16.30 \\
\hline & Average & 35.05 & 21.89 \\
\hline $\begin{array}{l}\text { Monthly Average Energy Consumption } \\
\text { Savings by Computer (kWh) }\end{array}$ & \multicolumn{3}{|c|}{$35.05-21.89=13.16$} \\
\hline Monthly Energy Consumption Savings for & \multirow{3}{*}{\multicolumn{3}{|c|}{$13.16 \times 615$ computers $=8,093$}} \\
\hline Central Office Computers with Active & & & \\
\hline Power Management (kWh) & & & \\
\hline Monthly Energy Consumption Cost Savings & \multirow{3}{*}{\multicolumn{3}{|c|}{$10,528 \mathrm{kWh} \times \$ 0.065=\$ 526.07$}} \\
\hline for Central Office Computers with Active & & & \\
\hline Power Management (\$) & & & \\
\hline
\end{tabular}

Results comparing computer energy consumption with and without power management over a 16-month period indicate a $37 \%$ decrease in energy consumption ( $8093 \mathrm{kWh}$ decrease) and related costs (\$526 savings per month) associated with implementation of a computer power management policy. These results over an extended period of time confirm initial short-term results and document the long-term success of the power management pilot. Power management policies have been activated in all spaces since 14 July 2014 (10 months). The 37\% decrease in energy consumption indicated during this research serve to corroborate results achieved by Agarwal et al., falling within the range of potential savings indicated during their studies (Agarwal et al, 2010) [7]. 


\section{Future Recommended Actions and Conclusions}

Given the aforementioned research progress and observations, TDEC recommended continued implementation of power management policies throughout TDEC Central Office in Tennessee Tower, and also recommended expanding implementation of power management policies throughout the remainder of TDEC offices, including all eight field offices and 56 state parks, where practicable and possible. Using savings achieved during the pilot, if computer power management policies were implemented on computers at TDEC offices across the state, energy cost savings could amount to an estimated \$16,937 annually (Assumes approximately 1650 TDEC computers capable of being managed by computer power management policies.). Beyond TDEC, based on the positive results of the CPM Pilot Project in a representative state department, it is recommended that power management policy implementation be expanded to computers across state executive agencies in Tennessee state government. If implemented throughout State of Tennessee executive agencies across the state, energy cost savings could amount to an estimated $\$ 323,341$ annually (Assumes approximately 31,500 computers statewide overseen by OIR and capable of being managed by computer power management policies.).

Implementation of computer power management policies present a significant cost saving opportunity for the public sector generally, not only State of Tennessee executive agencies. Similar schemes may prove an effective low- or no-cost way for public sector entities to reap energy and cost savings from reduced energy consumption, freeing up taxpayer dollars for provision of additional services. Work environments are rapidly evolving, and how technology is used within the workplace is experiencing similar changes. Therefore, beyond this current study, an area of future research may be examining cost-effective opportunities for reducing energy consumption of networked, mobile devices. With the proliferation of tablet computers, smartphones, and other technologies, the workplace is shifting to using energy in very different ways than it has historically. However, there still may be considerable opportunities for reducing energy consumption from those devices, too.

\section{Acknowledgments}

A special thanks to TDEC Information Services Division staff, specifically Talton Pettigrew, Skeet Owen, David Wesch, and Ken Bernhardt, and TDEC Office of Policy and Planning staff, specifically Michelle Walker Owenby, for support and assistance with this project. Gratitude is also extended to Scott Slusher and Matt Milam from TDEC's Office of Energy Programs for their input during pilot development. Appreciation is also extended to TDEC leadership, specifically Commissioner Bob Martineau, for his direction in initiating this project. State of Tennessee Office of Information Services likewise deserves mention for their willingness to allow TDEC to pursue this project.

\section{Author Contributions}

Kendra Abkowitz Brooks developed the concept for research and Talton Pettigrew investigated opportunities for using existing software for implementation. Talton Pettigrew also designed and administered the computer power management policies used throughout the research and was the collector of data. Kendra Abkowitz conducted all data analysis and calculations and documentation of research methodology and findings and preparation of manuscript. 


\section{Conflicts of Interest}

The authors declare no conflict of interest.

\section{References}

1. Abkowitz Brooks, K.; Pettigrew, T. Computer Power Management Pilot Proposal. 2014, unpublished work.

2. Abkowitz Brooks, K.; Pettigrew, T. Computer Power Management Status Report. 2015, unpublished work.

3. Pickavet, M.; Vereecken, W.; Demeyer, S.; Audenaert, P.; Vermeulen, B.; Develder, C.; Colle, D.; Dhoedt, B.; Demeester, P. Worldwide Energy Needs for ICT: The Rise of Power-Aware Networking. In Proceedings of the IEEE International Conference on Advanced Networks and Telecommunication Systems (ANTS), Bombay, India, 15-17 December 2008.

4. Average Retail Prices of Electricity. U.S. Energy Information Administration. Available online: http://www.eia.gov/totalenergy/data/monthly/pdf/sec9_11.pdf (accessed on 15 October 2015).

5. Alam, M.S.; Brooks, L. Identifying a Public Sector Information Systems (PSIS) for E-service: A Case of Land Records E-service in Bangladesh. Lect. Notes Comput. Sci. 2014, 8653, 106-119.

6. Alam, M.S.; Brooks, L. Designing an Information System for Updating Land Records in Bangladesh: Action Design Ethnographic Research (ADER). In Grand Successes and Failures in IT. Public and Private Sectors; Springer: Berlin, Germany, 2013; pp. 359-374.

7. Agarwal, Y.; Savage, S.; Gupta, R. SleepServer: A Software-Only Approach for Reducing the Energy Consumption of PCs within Enterprise Environments. In Proceedings of the UNISEX Annual Technical Conference, Boston, MA, USA, 23-25 June 2010.

8. Nedevschi, S.; Popa, L.; Iannaccone, G.; Ratnasamy, S.; Wetherall, D. Reducing Network Energy Consumption via Sleeping and Rate-Adaptation. In Proceedings of UNISEX Symposium on Networked Systems Design and Implementation, San Francisco, CA, USA, 16-18 April 2008.

9. Gunaratne, C.; Christensen, K.; Nordman, B. Managing energy consumption costs in desktop PCs and LAN switches with proxying, split TCP connections, and scaling of link speed. Int. J. Netw. Manag. 2005, 15, 297-310.

10. Microsoft System Center Technical Documentation Library. Available online: https://technet.microsoft.com/library/cc507089.aspx (accessed on 8 August 2015).

11. System Center 2012 R2 Configuration Manager. Available online: http://www.microsoft.com/ en-us/server-cloud/products/system-center-2012-r2-configuration-manager/ (accessed on 12 November 2013).

(C) 2015 by the authors; licensee MDPI, Basel, Switzerland. This article is an open access article distributed under the terms and conditions of the Creative Commons Attribution license (http://creativecommons.org/licenses/by/4.0/). 